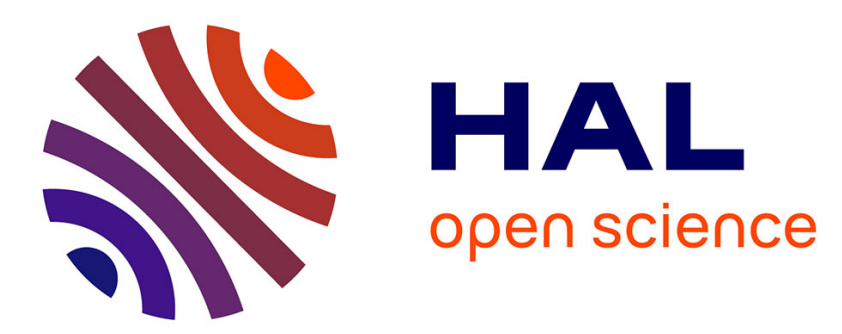

\title{
Green's Function Retrieval with Absorbing Probes in Reverberating Cavities
}

\author{
Matthieu Davy, Julien de Rosny, Philippe Besnier
}

\section{To cite this version:}

Matthieu Davy, Julien de Rosny, Philippe Besnier. Green's Function Retrieval with Absorbing Probes in Reverberating Cavities. Physical Review Letters, 2016, 116 (21), pp.213902. 10.1103/PhysRevLett.116.213902 . hal-01343529

HAL Id: hal-01343529

\section{https://hal-univ-rennes1.archives-ouvertes.fr/hal-01343529}

Submitted on 13 Mar 2017

HAL is a multi-disciplinary open access archive for the deposit and dissemination of scientific research documents, whether they are published or not. The documents may come from teaching and research institutions in France or abroad, or from public or private research centers.
L'archive ouverte pluridisciplinaire HAL, est destinée au dépôt et à la diffusion de documents scientifiques de niveau recherche, publiés ou non, émanant des établissements d'enseignement et de recherche français ou étrangers, des laboratoires publics ou privés. 


\title{
Green's function retrieval with absorbing probes in reverberating cavities
}

\author{
Matthieu Davy \\ Institut d'Electronique et de Télécommunications de Rennes, \\ UMR CNRS 6164, Université de Rennes 1, Rennes 35042, France ${ }^{*}$ \\ Julien de Rosny \\ ESPCI ParisTech, PSL Research University, CNRS, \\ Institut Langevin - 1 rue Jussieu, F-75005, Paris, France \\ Philippe Besnier \\ Institut d'Electronique et de Télécommunications de Rennes, \\ UMR CNRS 6164, INSA de Rennes, Rennes 35708, France.
}

(Dated: March 7, 2016)

\begin{abstract}
The cross-correlation of a diffuse wavefield converges toward the difference between the anticausal and causal Green's functions between two points. This property has paved the way to passive imaging using ambient noise sources. In this letter, we investigate Green's function retrieval in electromagnetism. Using a model based on the fluctuation dissipation theorem, we demonstrate theoretically that the cross-correlation function strongly depends on the absorption properties of the receivers. This is confirmed in measurements within a reverberation chamber. In contrast to measurements with non-invasive probes, we show that only the anti-causal Green's function can be retrieved with a matched antenna. Finally, we interpret this result as an equivalent time-reversal experiment with an electromagnetic sink.
\end{abstract}

The Green's function retrieval technique is now widely used for passive imaging from ambient noise. It is based on the cross-correlation of a diffuse wavefield with an array of receivers. The fluctuation-dissipation theorem (FDT) shows that the cross-correlation at two points of a field at thermal equilibrium is proportional in the frequency domain to the imaginary part of the Green's function between them $[1+5]$. This property has been extended to non-thermal noise sources for which the equipartition principle is fulfilled [4 9]. In the time domain, the anti-causal and causal Green's function are obtained so that the impulse response between two receivers can be reconstructed for sufficiently broadband signals. The Green's function can be reconstructed from thermal radiations in a cavity [4, 10 and for a diffuse field generated by uncorrelated random sources evenly distributed in a volume [7, 8, 11, 12. In chaotic cavities [4, 1315] and in random media [5, 16, 18, multiple scattering increases the convergence rate of the cross-correlation toward the Green's function with respect to the number of noise sources. The ballistic waves and the first echoes which are usually of interest for imaging purposes can therefore be estimated within disordered media even with a small number of noise sources.

The ambient noise correlation method has paved the way for spectacular results in seismology [19, 20, (see e.g. Ref. 21] for a review). It has also been demonstrated with acoustic [4, 22, 23], elastic [15] and electromagnetic waves in microwave [10] and optical frequency ranges [24].

Studies on Green's function retrieval have so far focused on non-invasive measurements of the field, which is for instance the case of seismic stations. This means that the wavefield is barely modified by the presence of the sensor. In this case, the cross-correlation of an equipartitioned field probed with point-like receivers is a symmetrical function in the time domain. In electromagnetism, efficient antennas are however absorbing and scattering receivers. For radio frequency waves, antennas are characterized by their internal impedance $Z_{11}$. The maximum power is extracted from the field when the receiving antenna is matched with the load impedance $Z_{L}, Z_{L}=Z_{11}^{*}$. In optics, the field can be probed with quantum dipoles or nanoantennas [25. An analog impedance concept is increasingly used to model the properties of these nano-devices (absorption, resonance ...) 26 28]. Those sensors may be used to measure the cross-density of states (CDOS) which characterizes the coherence of a wavefield independently of the source distribution [29]. The CDOS is proportional to the imaginary part of the trace of the Green's tensor between two points. Similarly to Green's function estimation, the CDOS can be obtained from the cross-correlation of an equipartitioned field between two positions [18. However the presence of these nano-devices may strongly modify the field and may therefore induce an important discrepancy from the theoretical predictions of the CDOS involving only the electromagnetic fields $\mathrm{E}$ and $\mathrm{H}$.

In this letter, we investigate the cross-correlation $C(t)=s_{1}(-t) \otimes s_{2}(t)$ of two signals $s_{1}(t)$ and $s_{2}(t)$ obtained by measuring an equipartioned field at two positions with absorbing receivers. We demonstrate using the FDT that probing the wavefield with antennas with different impedances gives an asymmetrical signal in the time domain. We show that in the case of a first matched 
antenna and a second non-invasive probe, $C(t)$ even vanishes at positive times and is proportional to the anticausal Green's function between them, $C(t) \sim G_{12}(-t)$. Those theoretical results are confirmed in measurements in a chaotic cavity. We take advantage of the diffuse field generated by a single source in a mode-stirred reverberation chamber (RC) to retrieve the impulse response between two receivers. Those results illuminate the central role of antenna absorption. Furthermore since the crosscorrelation can be interpreted as a time reversal (TR) process, we show that it implies that the incoming energy of a time-reversed wavefront is completely absorbed by a single matched antenna.

The FDT expresses the cross-correlation at frequency $\omega$ of two voltages $U_{1}$ and $U_{2}$ at thermal equilibrium in terms of the mutual impedance matrix $\mathbf{Z}$ between the ports [2, 30],

$$
C_{12}(\omega)=2\left[Z_{1,2}+Z_{2,1}^{*}\right] k_{B} T(\omega)
$$

Here $T(\omega)$ is the temperature of the system. This relation is an extension to multiport systems of the power spectral density of the Johnson noise measured by a resistor $R_{0}$ given by $4 R_{0} k_{B} T(\omega)$ [31]. For a reciprocal system, the real part of the mutual impedance matrix is obtained, $C_{12}(\omega)=4 k_{B} T(\omega) \operatorname{Re}\left\{Z_{2,1}\right\}$. This result not only holds for electrical systems, but also as soon as probes linearly convert a wavefield into quantities such as voltage/current for electromagnetic waves or force/material velocity for acoustic waves. It is of great interest for passive imaging since the cross-correlation of voltages at thermal equilibrium measured with two antennas in their open-circuit modes is similarly given by Eq. (1). The mutual impedance of two resonant antennas in electromagnetism is $Z_{2,1}=i \int \mathbf{n}_{2}^{T}\left(\mathbf{x}_{2}\right) \mathbf{G}\left(\mathbf{x}_{1}, \mathbf{x}_{2}\right) \mathbf{n}_{1}\left(\mathbf{x}_{1}\right) d^{3} \mathbf{x}_{1} d^{3} \mathbf{x}_{2}$ where $\mathbf{G}$ is the electric dyadic Green's function and $\mathbf{n}_{j}\left(\mathbf{x}_{j}\right)$ is the normalized distribution of the current within the antenna $j$. For two dipoles of lengths $l_{1}$ and $l_{2}$ small compared to the wavelength, $l_{1}, l_{2} \ll \lambda$, the FDT therefore writes

$C_{12}\left(\mathbf{x}_{1}, \mathbf{x}_{\mathbf{2}}, \omega\right)=4 l_{1} l_{2} k_{B} T(\omega) \frac{G\left(\mathbf{x}_{1}, \mathbf{x}_{2}, \omega\right)-G^{*}\left(\mathbf{x}_{1}, \mathbf{x}_{2}, \omega\right)}{2 i}$,

with $G\left(\mathbf{x}_{1}, \mathbf{x}_{2}, \omega\right)=\mathbf{n}_{2}^{T}\left(\mathbf{x}_{2}\right) \mathbf{G}\left(\mathbf{x}_{1}, \mathbf{x}_{2}, \omega\right) \mathbf{n}_{1}\left(\mathbf{x}_{1}\right)$. This equation is the classical expression of the crosscorrelation function for non-invasive pointlike probes [79 .

For absorbing antennas, the model of a system at thermal equilibrium cannot be used since the part of the energy that is absorbed by the antenna is not compensated by a corresponding immediate thermal emission. Eq. (1) can hence not be applied in its form. Nevertheless the correlation matrix $\mathbf{C}(\omega)$ can be obtained from the FDT using Thevenin's theorem 32]. The two ports system is equivalent of two noise voltage sources that are loaded with the mutual impedance matrix $\mathbf{Z}$. Fully taking into account the coupling between the ports yields [33],

$$
\mathbf{C}(\omega)=2 k_{B} T(\omega) \mathbf{Q}\left[\mathbf{Z}+\mathbf{Z}^{*}\right] \mathbf{Q}^{\dagger},
$$

where the matrix $\mathbf{Q}$ is given by, $\mathbf{Q}=\mathbf{Z}_{L}\left(\mathbf{Z}+\mathbf{Z}_{L}\right)^{-1}$. The load impedance $\mathbf{Z}_{L}$ is a diagonal matrix whose first and second elements are the load impedance of the two antennas $Z_{L 1}$ and $Z_{L 2}$, respectively. For non-invasive probes, the currents in the antennas are weak since $Z_{L 1}$ and $Z_{L 2}$ are much larger than the elements of $\mathbf{Z}$. This gives $\mathbf{Z}+\mathbf{Z}_{L} \sim \mathbf{Z}_{L}$ and $C_{12}(\omega)$ reduces to Eq. (1) as expected.

We now investigate the interesting case of the crosscorrelation between an absorbing antenna with internal impedance $Z_{11}$ and a non-invasive probe. Straightforward calculations using Eq. 3) yield,

$$
C_{12}(\omega)=2 k_{b} T(\omega) \frac{Z_{L 1}^{*}}{Z_{L 1}^{*}+Z_{11}^{*}}\left(Z_{1,2}^{*}+\Gamma Z_{1,2}\right) .
$$

Here $\Gamma=\left(Z_{L 1}-Z_{11}^{*}\right) /\left(Z_{L 1}+Z_{11}\right)$ is the usual reflection parameter due to the impedance mismatch between the load impedance $Z_{L 1}$ and the internal impedance of the first antenna. When the first antenna is matched, $Z_{11}^{*}=$ $Z_{L 1}(\Gamma=0)$, with a small reactive part $\left(\operatorname{Im}\left\{Z_{11}\right\} \ll\right.$ $\left.\operatorname{Re}\left\{Z_{11}\right\}\right), C_{12}(\omega)=k_{b} T Z_{1,2}^{*}$. Only the anti-causal Green's function between the antennas is hence retrieved. This phenomenon occurs because the power dissipated by the load impedance is equal to $k_{b} T(\omega)$.

The analogy with time reversal 34 provides an elegant way to interpret the result of the cross-correlation [16]. The cross-correlation of a diffuse field is indeed equivalent to a TR process in which the field emitted by the first antenna at $\mathbf{x}_{1}$ is time-reversed by the noise sources and the field is measured on a second antenna at $\mathbf{x}_{2}$. For noninvasive receivers, the time reversed field is the superposition of the converging wave proportional to $\mathbf{G}^{*}\left(\mathbf{x}_{1}, \mathbf{x}_{2}\right)$ followed by a diverging wave which is in opposition of phase $-\mathbf{G}\left(\mathbf{x}_{1}, \mathbf{x}_{2}\right), \psi_{T R}\left(\mathbf{x}_{2}\right) \sim \mathbf{G}^{*}\left(\mathbf{x}_{1}, \mathbf{x}_{2}\right)-\mathbf{G}\left(\mathbf{x}_{1}, \mathbf{x}_{2}\right)$. Their interference suppresses the singularity at the source and the focal spot has a width given by the diffraction limit. However when the initial source antenna is absorbing, a part of the incident energy is dissipated in the load in the second step of the TR process. Eq. (4) demonstrates that the time-reversed field $\psi_{T R}\left(\mathbf{x}_{2}\right) \sim$ $\mathbf{G}^{*}\left(\mathbf{x}_{1}, \mathbf{x}_{2}\right)-\Gamma \mathbf{G}\left(\mathbf{x}_{1}, \mathbf{x}_{2}\right)$ is not symmetrical in the time domain because of the virtual emission of an electric field proportional to $(1-\Gamma) \mathbf{G}\left(\mathbf{x}_{1}, \mathbf{x}_{2}\right)$ due to the current circulation within the antenna.

A matched antenna $(\Gamma=0)$ fully absorbs the energy of the incoming field and $\psi_{T R} \sim \mathbf{G}^{*}\left(\mathbf{x}_{1}, \mathbf{x}_{2}\right)$. This relation is identical to the "acoustic sink" model that shows that it is possible to focus waves with sub-wavelength resolution [35. Such a perfect absorption has also been shown 


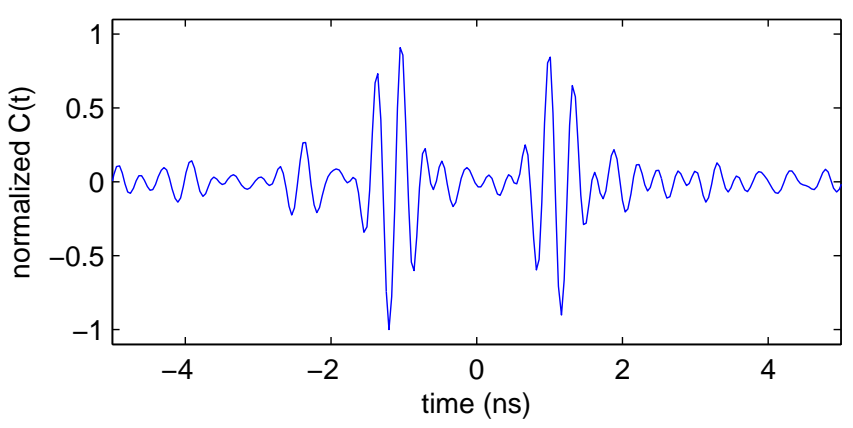

FIG. 1. Normalized cross-correlation $C(t)$ obtained from measurements of the field with a non-invasive electro-optical probe translated at two positions.

possible with a lossy object illuminated with a properly designed wavefront [36, 37. These results illustrate that a matched antenna acts as a coherent passive sink in a TR process. We emphasize that a perfect absorption can be detected in measurements only with a non-invasive probing of the time-reversed field. When a second absorbing antenna is used to probe the field at $\mathbf{x}_{2}$, the incoming wavefront can be strongly modified and is not the perfect time-reversed field of the source. The second pulse at positive times is then retrieved.

Those results are now confirmed in measurements in the microwave range. Measurements are carried out within a chaotic cavity, here a reverberation chamber (RC) of volume $V=93.3 \mathrm{~m}^{3}$. We aim to reconstruct the impulse response between two receiving antennas at locations $x_{1}$ and $x_{2}$ from the diffuse wavefield generated by a single source, a third antenna at $x_{3}$. The equipartitioned field is not generated from thermal radiations but from multiple scattering at the boundary of the chaotic cavity and satisfies an equipartition-like relation [38, 39]. We measure the transmission coefficients $s_{13}(\omega)$ and $s_{23}(\omega)$ with a network analyzer in the [2-4] GHz frequency band with steps of $\delta \mathrm{f}=100 \mathrm{kHz}$. This frequency range is well above the first mode resonance of the $\mathrm{RC}(43 \mathrm{MHz})$ so that the field is expected to be statistically isotropic, uniform and depolarized [40. The cross-correlation of the two signals is given by $C(\omega)=s_{13}(\omega) s_{23}^{*}(\omega) V_{0}^{2}(\omega)$ where $V_{0}(\omega)=V_{0}$ is the excitation voltage on port 3 .

The emitting antenna is located near a corner of the $\mathrm{RC}$ to reduce the contribution of the ballistic waves. To enhance the contribution of late arrivals, we compensate the exponential decrease of the envelopes of the signals in the time domain. The inverse Fourier transform of $s_{13}(\omega)$ and $s_{23}(\omega)$ are multiplied by $\exp \left(t / \tau_{a}\right)$, where $\tau_{a}=1.8 \mu \mathrm{s}$ is the average damping time of the $\mathrm{RC}$, and then cross-correlated. Despite the self-averaging property of the cross-correlation in reverberating media [41, $C(t)$ is dominated by strong spurious fluctuations because i) a single source is used; and ii) $\tau_{a}$ is small compared to the Heisenberg time of the cavity, $\tau_{H} \sim 8 \mathrm{~ms}$, which is estimated from the modal density per Hertz given by Weyl's formula. We then average the crosscorrelation over fifty positions of a stirrer made of 6 aluminium blades with surfaces of $\sim 750 \lambda^{2}$. Its rotation provides statistically independent realizations of the diffuse field [42] so that we expect that $C(t)$ converges toward the average Green's function of the RC consisting of the direct wave between the antennas and the first echoes that are not scattered by the stirrer. In Eq. (1), $Z_{2,1}$ must therefore be replaced by its average over the positions of the stirrer, $\left\langle Z_{2,1}\right\rangle$. This change is implicit in the following analysis of the measurements.

The cross-correlation $C(t)$ is first measured by translating at two positions an electro-optical probe of length of order of a tenth of the wavelength. The probe allows a non-invasive measurement of the field. Its translation barely modifies the diffuse field within the chamber so that $C(t)$ is equivalent to the cross-correlation recorded with two similar probes. $C(t)$ is seen in Fig. 1 to be a symmetrical function consisting of two pulses at $-1.2 \mathrm{~ns}$ and $1.2 \mathrm{~ns}$, in agreement with Eqs. (1)-(2).
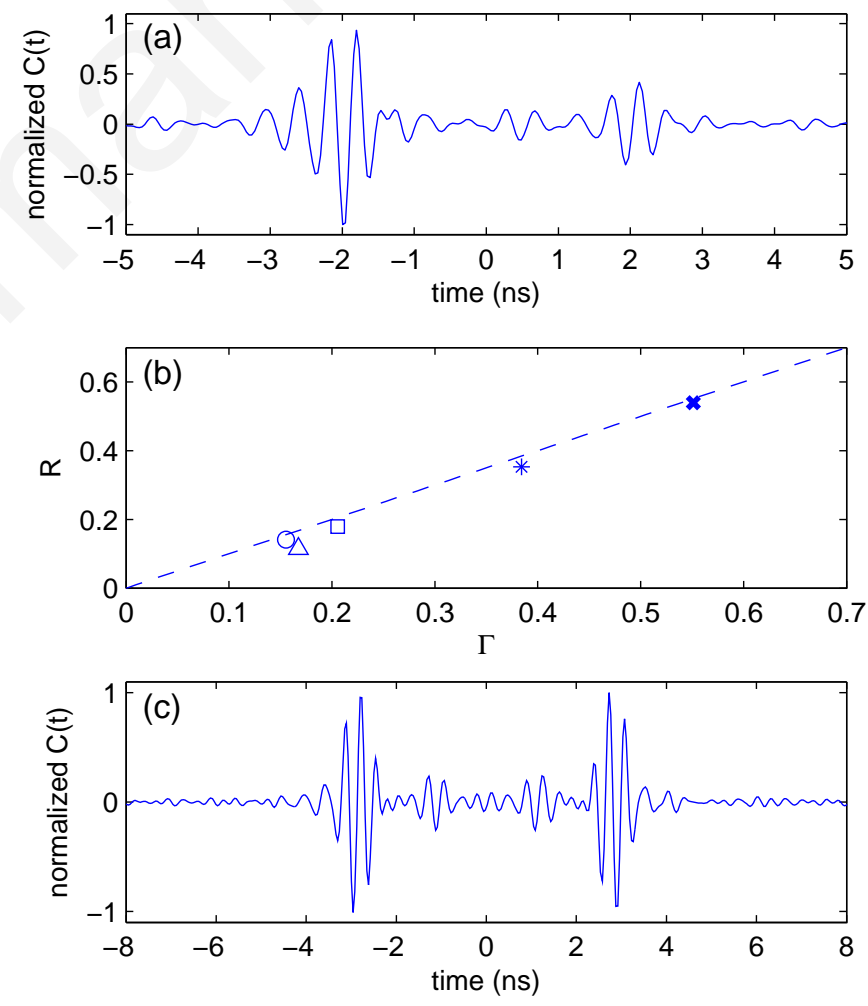

FIG. 2. (a) Normalized cross-correlation $C(t)$ for a horn antenna and a non-invasive probe. (b) Ratio of the maximum amplitude at negative times and maximum amplitude at positive times with the impedance mismatch $\Gamma$ for a UWB horn antenna (star), two other horn antennas (circle and cross), a discone antenna (square) and a log-periodic dipole antenna (triangle). The straight line is $R=\Gamma$. (c) Normalized crosscorrelation $C(t)$ for the same receiving horn antennas facing each other. 
The cross-correlation is then performed between a horn antenna and the probe with the same polarization. A strong asymmetry is seen in Fig. 2a despite the isotropic nature of the wavefield within the cavity. This result may seem surprising at first glance regarding previous works which mainly consider non-invasive receiver since a strong asymmetry usually reveals a non-uniform spatial distribution of the field [20, 43, 44] which is definitely not the case here. However this asymmetry is fully predicted by Eq. (4). It shows that the amplitude of $C(t)$ at positive times relative to its amplitude at negative times, $R=\max [|C(t>0)|] / \max [|C(t<0)|]$, is expected to be equal to $\Gamma$. $\Gamma$ can be evaluated from the $S_{11}$ parameter $\left(\Gamma=\left|S_{11}\right|\right)$. From Fig. 2a, we find $R=0.35$ which is in good agreement with the average over the bandwidth of $\Gamma=0.38$. $R$ and $\Gamma$ are then measured for other antennas (a horn antenna, a discone antenna and a log-periodic dipole antenna) in various frequency ranges and are seen to be close in Fig. 2b. We find ratios $R$ typically slightly smaller than the theoretical predictions because Eq. (4) does not include losses which are $\sim 10 \%$ for those antennas.

We also compute the cross-correlation for two similar horn antennas facing each other in Fig. 2c. The symmetry is obtained as for non-invasive probes but is now related to the use of similar scattering and absorbing antennas. More generally, it can be shown using a computer algebra system that the ratio of the amplitudes at positive and negative times is given by the ratio of the impedance mismatches of the two antennas, $\Gamma_{1} / \Gamma_{2}$. Note that retrieving the Green's function with those aperture antennas was not trivial regarding previous studies con-
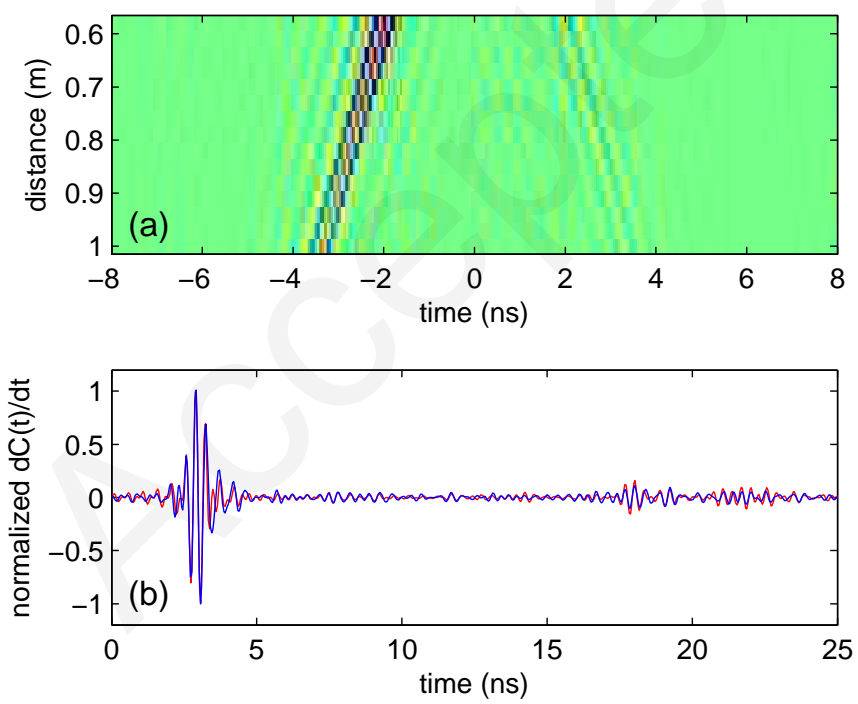

FIG. 3. (a) Colormap representation of $C(t)$ when the distance between the horn antenna and the probe increases from $0.55 \mathrm{~m}$ to $1 \mathrm{~m}$. (b) $d C(t) / d t$ (blue) taken at negative times is compared to the impulse response (red).

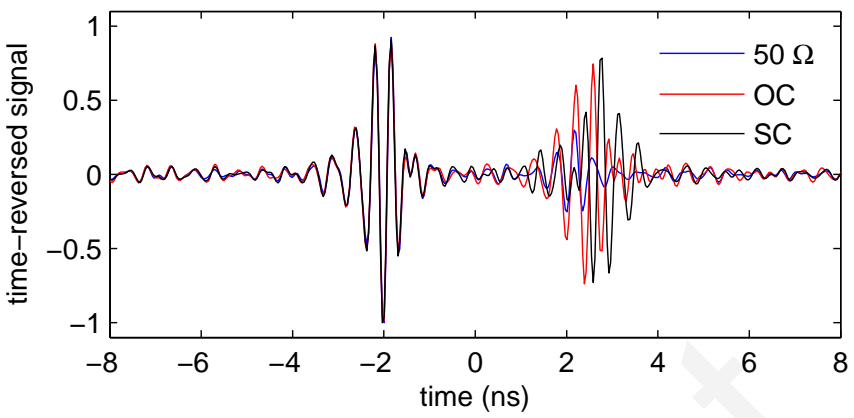

FIG. 4. The time-reversed signal is plotted for three load impedances: a $50 \Omega$ load (blue), an open-circuit load (red) and a short-circuit load (black).

sidering non-invasive receivers. Noise sources that contribute to the cross-correlation have indeed been shown to be in the stationary phase region [20, 43, 44] that is located in the alignment of the receivers. However, those contributions are weak for horn antennas because of their directivity patterns. The coupling between antennas is the key to interpret $C(t)$.

We show in Fig. 3 that we accurately retrieve the impulse response averaged over the positions of the stirrer between two receivers, here a horn antenna and the probe. The delay time of the two ballistic pulses is seen in Fig. 3a to correspond to the travel time between the two receivers. The normalized derivative of the crosscorrelation $d C(t) / d t$ at negative times is seen in Fig. 3b to be in very good agreement with the impulse response over more than $25 \mathrm{~ns}$. Not only the direct impulse response but also the first echoes in the cavity that are of interest for imaging applications are retrieved as seen around $18 \mathrm{~ns}$.

To further illustrate the influence of absorption in the load, we first measure the impulse response between the first horn antenna and the third antenna. We then modify the load impedance at the connector of the source antenna in the second step of a TR experiment. The timereversed field is measured with the non-invasive probe. We successively add a $50 \Omega$ load which is almost matched to the antenna internal impedance, an open-circuit (OC) load and a short-circuit (SC) load. The causal part of the time-reversed signal is seen in Fig. 4 to be strongly enhanced for the OC and SC loads $(|\Gamma| \sim 1)$ in comparison to the $50 \Omega$ load $(\Gamma \sim 0)$. The signals corresponding to the scattering from the $\mathrm{OC}$ and $\mathrm{SC}$ loads are in phase opposition 45. This confirms that the decrease of the signal at positive times is due to absorption by the load.

In conclusion, we have demonstrated Green's function retrieval in electromagnetism within a chaotic cavity with a single source. By modifying the modes of the cavity with a stirrer, the cross-correlation of the wavefield converges toward the average impulse response between the receivers. The theoretical and experimental results 
provide a new insight into passive imaging experiments using two absorbing receivers. They illuminate the relations between cross-correlation of random wavefields and scattering, absorption and impedance properties of the receivers. Our approach also provides a new framework for applications such as antenna directivity patterns measurements. In particular, it makes it possible to measure the coupling between two antennas that can be used in their receiving or transmitting modes only.

* matthieu.davy@univ-rennes1.fr

[1] S. M. Rytov, Y. A. K. Kravtsov, and V. I. Tatarskii, Principles of Statistical Radiophysics. 3: Elements of random fields (Springer, New York, 1989).

[2] M. L. Levin and S. M. Rytov, A Theory of Equilibrium Thermal Fluctuations in Electrodynamics (Nauka, Moscow, 1967).

[3] G. S. Agarwal, Phys. Rev. A 11, 230 (1975).

[4] R.L. Weaver and O.I. Lobkis, Phys. Rev. Lett. 87, 134301 (2001)

[5] B.A. van Tiggelen, Phys. Rev. Lett. 91, 243904 (2003)

[6] R. L. Weaver and O. I. Lobkis, J. Acoust. Soc. Am. 109, 2347 (2001).

[7] K. Wapenaar, Phys. Rev. Lett. 93, 254301 (2004)

[8] R. Snieder, Phys. Rev. E 69, 046610 (2004).

[9] K. Wapenaar, E. Slob, and R. Snieder, Phys. Rev. Lett. 97, $234301(2006)$.

[10] M. Davy, M. Fink, and J. de Rosny, Phys. Rev. Lett. 110, 203901 (2013)

[11] K. Sabra, P. Roux, and W. Kuperman, J. Acoust. Soc. Am. 118, 3524 (2005)

[12] P. Roux, K. G. Sabra, W. A. Kuperman, and A. Roux, J. Acoust. Soc. Am. 117, 79 (2005)

[13] O. Lobkis and R. Weaver, J. Acoust. Soc. Am. 110, 3011 (2001)

[14] R. L. Weaver and O. I. Lobkis, J. Acoust. Soc. Am. 118, 3447 (2005).

[15] L. Chehami, J. D. Rosny, C. Prada, E. Moulin, and J. Assaad, IEEE Trans. Ultrason. Ferroelectr. Freq. Control 62, 1544 (2015).

[16] A. Derode, E. Larose, M. Campillo, and M. Fink, Appl. Phys. Lett. 83, 3054 (2003)

[17] E. Larose, L. Margerin, A. Derode, B. van Tiggelen, M. Campillo, N. Shapiro, A. Paul, L. Stehly, and M. Tanter, Geophys. 71, SI11 (2006).
[18] J. De Rosny and M. Davy, Europhys. Lett. 106, 54004 (2014).

[19] N. Shapiro, M. Campillo, L. Stehly, and M. Ritzwoller, Science 307, 1615 (2005)

[20] M. Campillo and A. Paul, Science 299, 547 (2003)

[21] M. Campillo, P. Roux, B. Romanowicz, and A. Dziewonski, Treat. Geophys. , 256 (2014).

[22] P. Roux, W. Kuperman, and N. Grp, J. Acoust. Soc. Am. 116, 1995 (2004)

[23] T. Nowakowski, L. Daudet, and J. de Rosny, J. Acoust. Soc. Am. 138, 3010 (2015).

[24] A. Badon, G. Lerosey, A. C. Boccara, M. Fink, and A. Aubry, Phys. Rev. Lett. 114, 023901 (2015).

[25] L. Novotny and N. Van Hulst, Nature photon. 5, 83 (2011).

[26] A. Alù and N. Engheta, Phys. Rev. Lett. 101, 043901 (2008).

[27] J.-J. Greffet, M. Laroche, and F. Marquier, Phys. Rev. Lett. 105, 117701 (2010)

[28] R. L. Olmon and M. B. Raschke, Nanotechnology 23, 444001 (2012).

[29] A. Cazé, R. Pierrat, and R. Carminati, Phys. Rev. Lett. 110, 063903 (2013)

[30] R. Twiss, J. Appl. Phys. 26, 599 (1955).

[31] R. Dicke, Rev. Sci. Instrum. 17, 268 (1946)

[32] H. A. Haus and R. B. Adler, Circuit theory of linear noisy networks (Technology Press/Massachusetts Institute of Technology/John Wiley and Sons, Inc., NY, 1959).

[33] See Supplemental Material.

[34] M. Fink, Phys. Today 50, 34 (2008).

[35] J. de Rosny and M. Fink, Phys. Rev. Lett. 89, 124301 (2002).

[36] A. Sentenac, P. Chaumet, and G. Leuchs, Opt. Lett. 38, 818 (2013).

[37] H. Noh, S. M. Popoff, and H. Cao, Opt. Expr. 21, 17435 (2013).

[38] R. L. Weaver, J. Acoust. Soc. Am. 71 (1982).

[39] R. L. Weaver, J. Acoust. Soc. Am. 110 (2001).

[40] D. Hill et al., IEEE Trans. Electromagn. Comp. 40, 209 (1998).

[41] G. Bal, G. Papanicolaou, and L. Ryzhik, Stoch. Dyn. 2, 507 (2002)

[42] C. Lemoine, P. Besnier, and M. Drissi, IEEE Trans. Electromagn. Compat. 50, 227 (2008).

[43] R. Snieder, J. Sheiman, and R. Calvert, Phys. Rev. E 73, 066620 (2006).

[44] Y. Fan and R. Snieder, Geophys. J. Int. 179, 1232 (2009)

[45] R. Collin, IEEE Antennas Propag. Mag. 45, 119 (2003) 